\title{
$\mathfrak{I} \mathfrak{n} \mathfrak{h} \mathfrak{a} \mathfrak{l} \mathfrak{t}$
}

\section{Eriter Iheil.}

Die feftliđe Sälfte bes Sirdjenjabreș.

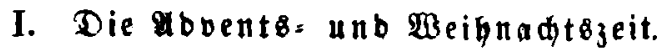

Erfie aboent8rode.

Geite

1. 1. (1. Abvent8jonntag.) Belobet (ei ber ba lommt in bem Ramen bes Serrn. PF. 118, 26.

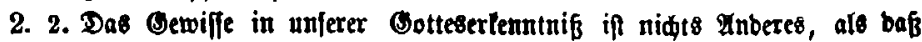
(3ott bie siebe fei. 1. Эob. 4, 16. . . . . . . . . . . . . 5

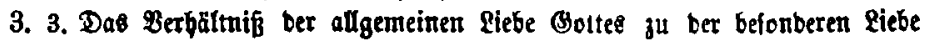
um Eptriti willen. 1. Diof. 1,31. . . . . . . . . . . . . 7

4. 4. 3 Bir follen uns leine faljde Borftellungen von ber Beredtigleit (Bottes, wie von feinem Dejen liberbaupt maden. Bj. 11, 7. . . . . . .

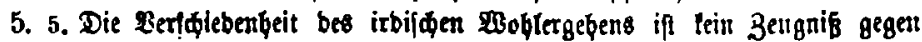
bie Ceredigleit Bottes. Sprildw. 22, 2. . . . . . . . . . 12

6. 6. Die Beredtigleit (Sottes offenbart fí barin, baß̉ allen biejelben Şülfo.

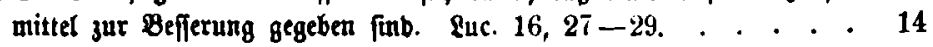

7. 7. Bott offenbart fíl in ber Ratur als cin (sont ber Dibnumg unb bes friebens. 1. (5or. 14, 33. . . . . . . . . . . . . . 16

3weite aboent8wode.

8. 1. (2. Mboent fonntag.) (bott bat in ber fidtbaren Sфippinng Ifem ein Mté gejeţt. ફio6. 38, 11.. . . . . . . . . . . . . 18

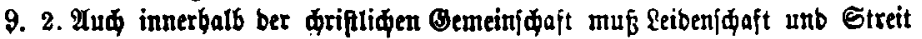
nad Botten Biflen Diaf balten. Mattb. 16, 18. . . . . . . 21

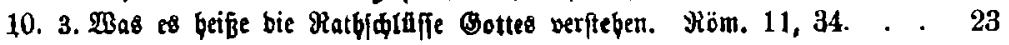

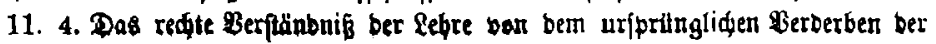
menfíliden Ratur. Matth. 13,23 . . . . . . . . . . 25

12. 5. Bon bar Erbfinte. Möm. 5, 19. . . . . . . . . . . . . 27

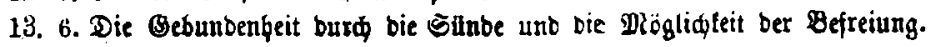
Datth. 8, 29. . . . . . . . . . . . . . . . . . . . 28

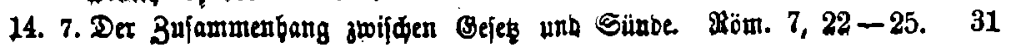


Sinbalt.

Dritte abont8wode.

15. 1. (3. Mbontsfonntag.) Die Bemeinfoft mit ben Werten ber fin. fternif. Epf. 5, 10.11. . . . . . . . . . . . . . . . 34

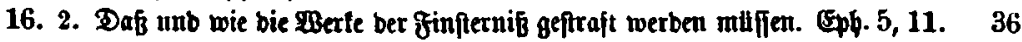

17. 3. Die Rotbwentigleit unb ber Segen bes gegenjetigen Befenntmifies ber Sllube unb gemeinfamer Fürkitte. 3ac. 5, 16. . . . . . . . 33

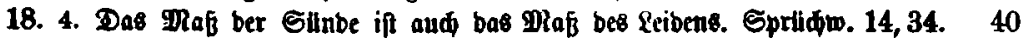

19. 5. Das leiben foll nidt zux Offenbarung ber Slinbe bienen. 1. Betr. 2, 23 . 41

20. 6. Bor bar Erideimung Ghrifti war eitte Beil ber Unwiffent. Apoftelg. 17, 30.43

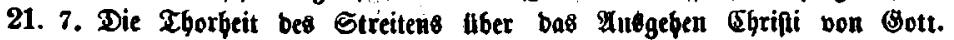
3०५. 16, 28. . . . . . . . . . . . . . . . . 46

\section{Bierte about onode.}

22. 1. (4. Mbventsfonntag.) Die menldlide Erideinung (5brifti ift bie

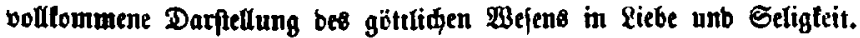
306. 14, 9. . . . . . . . . . . . . . . . . . . .

23. 2. Die redte Erfenntní̧ bes Erlöjers gelt nur aus ber vereinigten $B e$ -

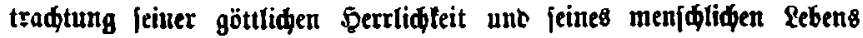
berbor. Ұob. 14, 10 . . . . . . . . . . . . . . .

24. 3. Cbriftus mafite von jeiner Beburt an ber jünbloje Sobn Bottes fein. \&uc. 1, 31. 32. . . . . . . . . . . . . . . . . . .

25. 4. Mur auf bem geraben $23 e g e$ ber Babrbeit tann bem Erlöjer ber Ein= 3ug in bie Đerzen bereitet werben. Suc. 3, 4. 5. . . . . . . .

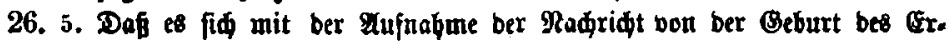
löjers äbnliø verbält wie in anbern men|ll. Dingen. \&uc. 2, 17-19.

27. 6. Die birten bei ber Beburt bes Exröjer8. \&uc. 2, 15. 17. 20. . . . 58

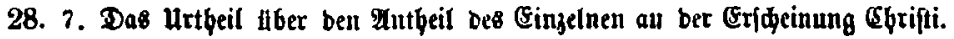
1. 3०4. 4, 2. 3 .

\section{Die Beignadt wode.}

29. 1. (Erfter $\mathfrak{B}$ eignadtieiertag.) (5ebet. Wie bie Feier ber Beburt bes Sobnes Bottes jebe äbnlide feiur ilbertrifft. Bat. 4, 4. . . .

30. 2. (3weiter Weibradt8feiertag.) Der Bujammenbang jwijden

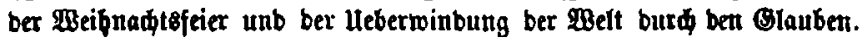

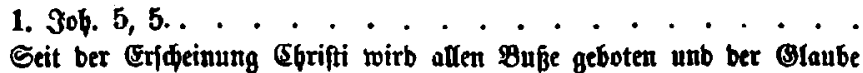
vorgebalten. Tpoftelg. 17, 30. 31.. . . . . . . . . . . . 67

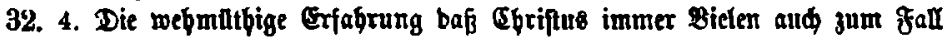
gereiфt. Euc. 2, 34. . . . . . . . . . . . . . . . . 69

33. 5. Der beţlebemitiłde Sinbermorb. Matth. 2, 16. . . . . . . . 71

34. 6. Die Fludt nad Egypten. Wlattb. 2, 14. . . . . . . . . . 74

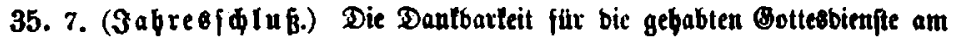

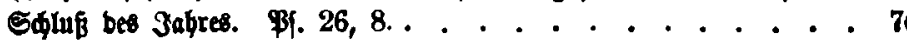




\section{Die Rentahrozeit.}

Erfte $\mathfrak{B o d y e . ~}$

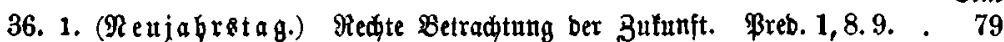

37. 2. Der Meujabromunfda: Friebe fei allen Bölfern! 304. 20, 19. . . 81

38. 3. Friebe fei ben Gemeinden in iȟrem gegeni. Berbältnip. 1. (Eor. 13, 4. 7. 83

39. 4. Friebe jei ben (Semeinben in ifrer eigenen Mitte. 1. Sor. 3, 21. . . 85

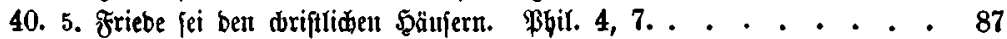

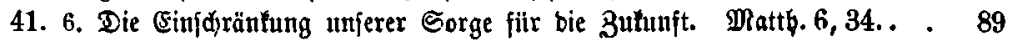

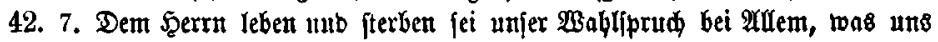

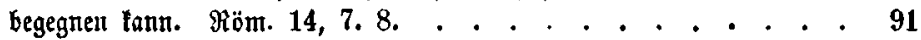

3 weite $\mathfrak{B o d y e .}$

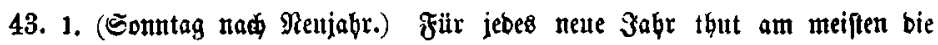
Siebe noth. நebr. 10, 24. . . . . . . . . . . . . . . 94

44. 2. Die Trette und ị̂r \&ohn. Matth. 25, 21. . . . . . . . . . 96

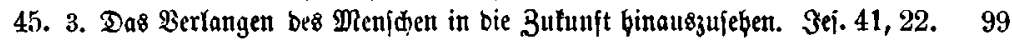

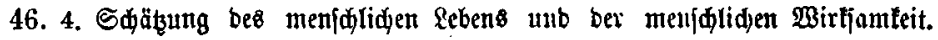
Bal. 2, 20. . . . . . . . . . . . . . . . . . . . 101

47. 5. Die Beurtbeilung ber Zeiden ber Beit. $\mathfrak{P a t t h}_{1}$ 16, 3. . . . . . 103

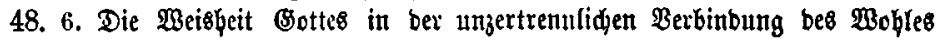

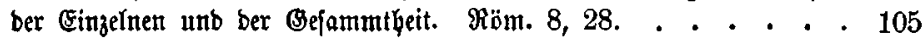

49. 7. Das Tradtent nad Dem Meide Botter. Matth. 6, 33. . . . . . 107

\section{Die Epiphaniazeit.}

\section{Erfte Bode. (1. Sonntag naథ Epiphanias.)}

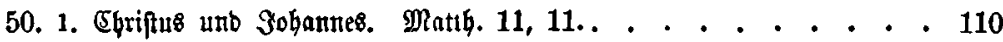

51. 2. (Ghriftus verfudt wie wir bow ohne Silnbe. Şebr. 5, 15. . . . . 112

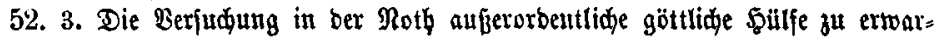
ten. Mattl. 4, 4. . . . . . . . . . . . . . . . . . 115

53. 4. Die Berfuфuıtg por ber Welt zu gläızen. Matth. 4, 7.. . . . . 117

54. 5. Die Berfudung Bemeinfdjaft mit bem Böjen zu guten 3weden jut

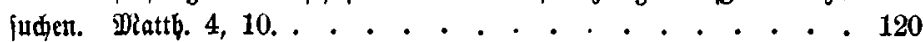

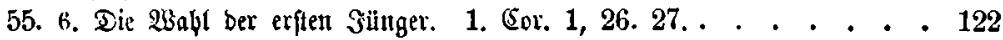

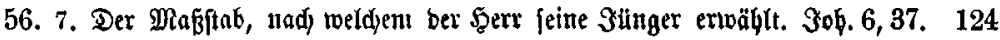

\section{3weite $2 B o d e . \quad$ (2. Sountag nad Epiphanias.)}

57. 1. \$ie bie natürlide \&ieke zar $\mathfrak{B a b r b e i t ~ f i d ~ m i t ~ b e m ~ a l l g e m e i n e n ~} \mathfrak{B e r}=$ berben ber mentjhlidjen $\mathfrak{R a t u r}$ verträgt. Эoh. 1, 47. . . . . . .

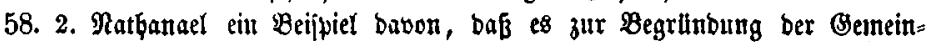

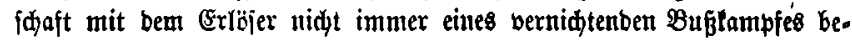

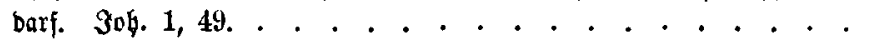

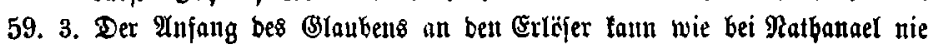

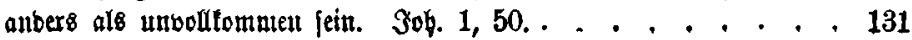


60. 4. Die Arbeit beg Đerm an feitent 3angern. 3of. 15, 16. . . . . 133

61. 5. Die qiebe bes Eriöjers zu jeinen 3üngern. Sob. 13, 1. . . . . . 135

62. 6. Die शBiffamt. b. Erröjers im Grofen unb im Eimelnen. Matth. 4, 23. 137

63. 7. Die भrt mie ber Erlöjer mit Eiızeltren eine Bemeinjфaft anfnilpft. Matth̆. 10, 41. . . . . . . . . . . . . . . . . . 139

\section{Dritte Wode. (3. Sonntag nad (5ipbanias.)}

64. 1. Der Erlöjer mintt mur in 3irael. Möm. 15, 8. . . . . . . . 141

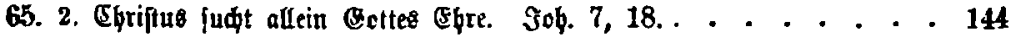

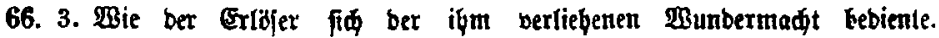
Mattb. 4, 23. . . . . . . . . . . . . . . . . . . . 146

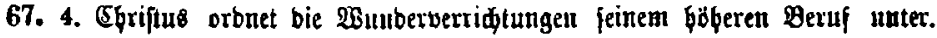
Ipoftelg. 10, 36-38. . . . . . . . . . . . . . . . . 148

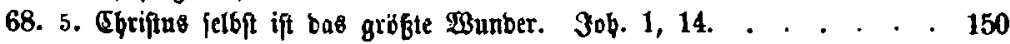

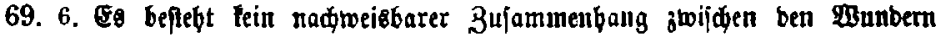
(Egrifti unb bem Glauben an ił̧n. Mattb. 9, 5. . . . . . . . 152

70. 7. Sbrift18 felbft unb bie Sdyrift neijent uns nidt auf jeine ben redtelt Brutto bes Blaukene, Apoftelg. 2, 22. 25. . . . . . 154

Bierte 2 ode. (4. Sonntag na旡 Epipbanias.)

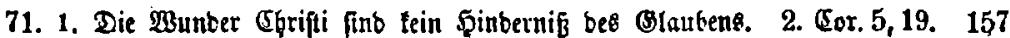

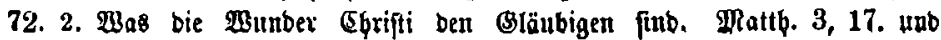
3o6. 14, 12. . . . . . . . . . . . . . . . . . . . 159

73. 3. Der Extöfer unb bie Geinigen fontern fid uidt won ber $\mathfrak{B e l t}$ ab. Gal. 5, 1. . . . . . . . . . . . . . . . . . . . 161

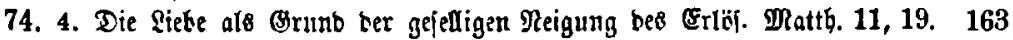

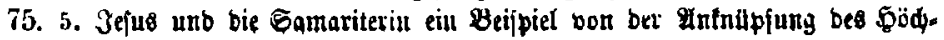
ften an Gewöbnlides aud im gefelligen \&ebent. 304.4,7.24. 26. . . 166

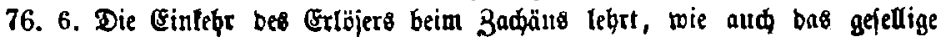

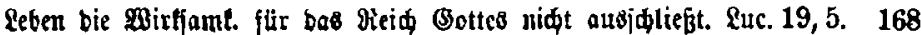

77. 7. Ebriffi 5erablafiung ju ben Beradteten bes Bolts. Suc. 19, 10. . . 170

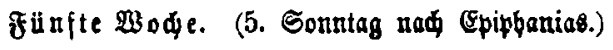

78. 1. Der Ernjt unb bie Milbe bes Ertäjers in jeinem gejelligen Umgange. Matth. 12, 20. . . . . . . . . . . . . . . . . . .

79. 2. Das Berbalten bes Erlöjer gegen bie $\mathfrak{B a n t e l m u l t b i g e n ~ u n b ~ b i e ~ f o i n t e ~}$ bes Buten. Matth. 9, 13. . . . . . . . . . . . . . . 175

80. 3. Das Berbatten Erlöjers gegen bie Samariterin, eine Beftätigung

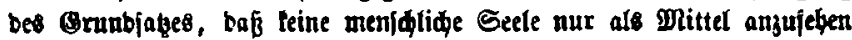
ift. 3ob. 17,6. . . . . . . . . . . . . . . . . . . 177

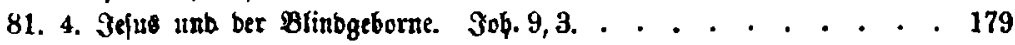

82. 5. 3eju unb bie Stumberin ober bas BerbäItniß zwijळen Bergebung unb Siebe. Surc. 7, 47. . . . . . . . . . . . . . . . . . 182

83. 6. Dex 他lafeube Эefus. Matth. 8,23.24. . . . . . . . . . 184

84. 7. Der Aleinglaube ber Jitnger. Datth.8,25. . . . . . . . . 186 


\section{Seф te 20 o. (6. Sountag naф Epiphaniad.)}

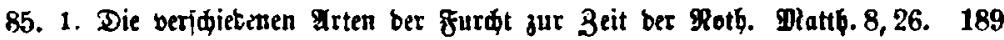

86. 2. Der Erlö|er madit teinen פnfprud auf bie Dantbarteit ber DRenjuen. Ruc. 17, 17. .

87. 3. Die Beprafung bee Petrus burd ben Erlöjer wegen falfфer Bejorg. niß̧ ber Riebe. Matth. 16, 22. 23. . . . . . . . . . . . . 193

88. 4. Die mittheilende liebe bes Grib̈fers. \$oధ̆.13,34.. . . . . . . 195

89. 5. Die bienenbe Riebe bes Erlijerb. Mattb. 20, 28. . . . . . . . 197

90. 6. Warun fo Biele gleid ben Gergejentern ben Erlöjer nidgt anjnebmen mollen. Mattb. 8,34 .

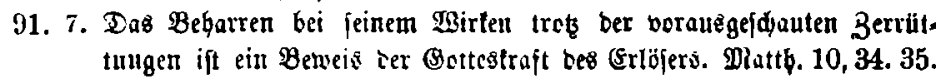

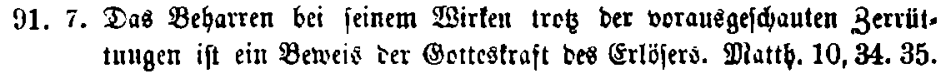

202

Sicbente 23 ode. (Sommtag Septuagefimii.)

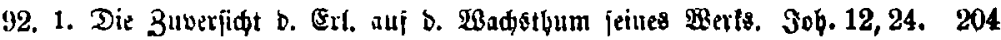

93. 2. Ebriftu ber einige redte Prophet. \&uc. 24,19. . . . . . . . 207

94. 3. Gluriftus ber cinige wahre Brefetgeber. 3er.31,33. . . . . . . 209

95. 4. Shriftus bir einige nabre jobepricfter. \$ebr.4, !č... . . . . 211

96. 5. (5priftus ber cinige wahre Hrebiger be friebens. 4pofterg. 10,36. . 214

97. 6. Sbriftut unjer Sorbilb. 1. Petr. 2, 21. . . . . . . . . . . 216

98. 7. Ģriftus forbert von ben Seinigen Inerfennung jeiner aufidlięliden

Würbe. $̧ 06.9,35-38$. . . . . . . . . . . . . . . 218

Adte Bode. (Somntag Sez̧agefimä.)

99. 1. Son ber falịden Galufternietrigung. Matth. 23, 12. . . . . . 220

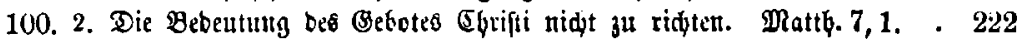

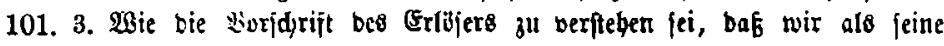
3ünger unịere gädyften bajien joflen. Kuc. 14,26. . . . . . . 225

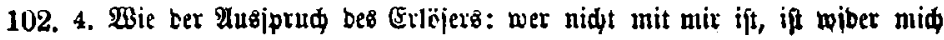
unb Dex entgegengejetzte zll vereinigen finb. \&uc. 11, 23, a. Marc. 9, 40. 227

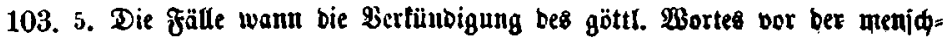

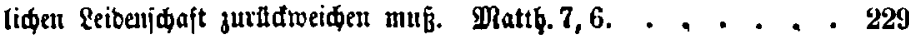

104. i. Wie von bem Tradten nad bem Reiфe Gottes aus bie Boriqrift bes Erlöjers aufjufaffer jei, bas roir Ænbern thun was wir moflen Dá̧ fie uns thun. Dlatth. 7, 12 . . . . . . . . . . • . .

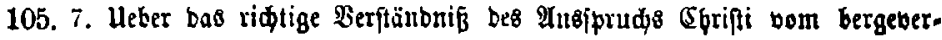
jebenten Blauben. Mattb. 17,20.. . . . . . . . . . 234

Rellite $\mathfrak{B} \circ$ de. (Sonntag Eftomili.)

106. 1. Was unter ben unnitzen Gorten ju verftegen joi, von benen wir Viechenldajt ju gebent baben. Miatţ̧. 12,36. . . . . . . . . . 236

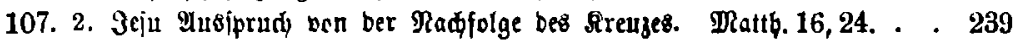

108. 3. Welden 2 Bertb bie Weifiagurgen bes alten Teftamentes itb. bie einjelneu Qeibelisumitünbe des Erlöjers baben. Darc. 9, 12. . . . . . . 241

109. 4. Das Bifien und bie Beifjagung bes Eribjers von jeinen eigenten Reiben. Matţ. 16,21. . . . . . . . . . . . . . 244 
110. 5. Strifti 3 eiffagung bon ben Reiben ber Seinigen. Matth. 23,34. . 246

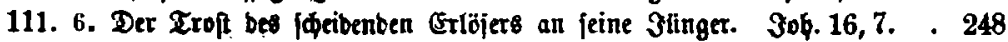

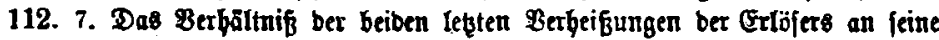
3unger bon feiner geiftigen Begenwart unb ber Senbung bee beiligen Beittes. Dratţ̨. 28, 20. unb 3oh. 14, 16. 17. . . . . . . . .

\section{Die $\mathfrak{B a}\{f i o n s z e i t$.}

\section{Erfte B̧ode. (Somntag Jubocabit.)}

113. 1. Das Beugn. Seju von fidj jelbf b. Beginu feiner Reiben. Mattb. 26, 55. 253

114. 2. EBie wenig ber $\mathbb{T}$ ob, ben er erleiben follte, für bent Errofer felbft be. beutete. 3ok. 16, 28. . . . . . . . . . . . . . . . . 255

115. 3. $9 \mathrm{~m}$ Beborjam gegen Bottes Befez unb Ratl gebt ber Extöjer jeinem

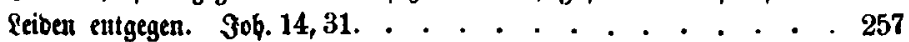

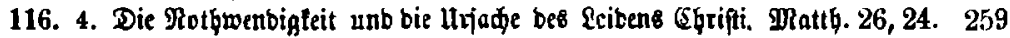

117. 5. Warum es notignentig war, bak ber Grlöjer fo zeitig ferben mujte. Eut. 12,49. . . . . . . . . . . . . . . . . . . 262

118. 6. Besbalb ber Errbjer eines gemaltjamen Tobes fterben muß̈te. 1. (5or. 1, 23. 24. . . . . . . . . . . . . . . . . . . . 263

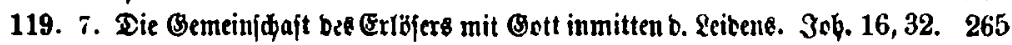

\section{3weite $\mathfrak{B}$ ode. (Sonntag Reminifcere.)}

120. 1. In nutefern bes Erlb̈ers geisen eine llekerwinsung ber $\mathfrak{B e l t}$ fei. Үol. 16, 33. . . . . . . . . . . . . . . . . . . . 268

121. 2. Die Cinfamteit in Reiben. 3o6. 16,32.. . . . . . . . . . 270

122. 8. Bon bem Bujammenbange zniiden bem Tobe bes Erröjers unb bem Glanben an ibn. quc. 24, 26. . . . . . . . . . . . . 272

123. 4. Det Tob Chrifti if bas einjige wabrbajte Bebüdtning ber Sánbe. หöm. 8, 3. . . . . . . . . . . . . . . . . . . . 275

124. 5. Xud wir milfen gleid wie Eqtiftus um ber Slinbe niflen reiben. Matth. 20, 22. . . . . . . . . . . . . . . . . . . 277

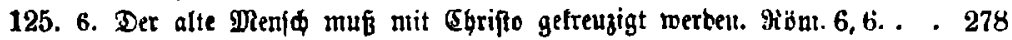

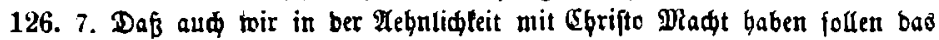
\&eber zи Іаffen. 30 . 10,18. . . . . . . . . . . . . . 280

\section{Dritte Wode. (Sonntag Dculi.)}

127. 1. Das Eigentbilmliđje in ben lez̧tet Leiben bes Erläfers non ber (Ge= jungesıebmung an. Ifpofterg. 2, 23. . . . . . . . . . . . 283

128. 2. Der Tob bes Erläpers als bie bollommenfte Dffenbarung feines Ges borjam gegen feinen bimmlifđen Pater. Patţ. 26,39. . . . 285 
Eeite

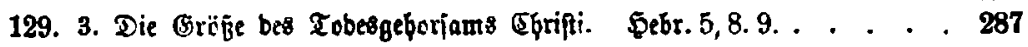

130. 4. Die (Sejangemebmung. Suc. 22,52.53. . . . . . . . . 290

131. 5. Die Unterntirfiglett bes Erlöjers gegen bie Obrigleit in jeinen Reiben. §ob. 18, 11. . . . . . . . . . . . . . . . . . . . 292

132. 6. Die Freimilthigfeit bes Erröjerళ gegen bie Dbrigleit. \&uc. 19, 42.. . 294

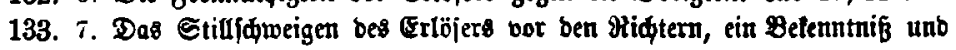

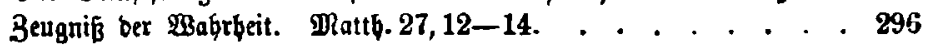

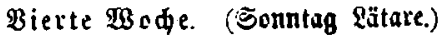

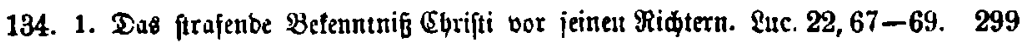

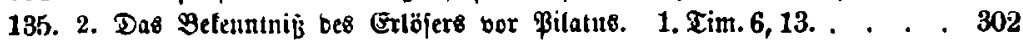

136. 3. Bei ber Berurtbeihung bes Ertöjers war fein \&eiben niфht ber göttlidje

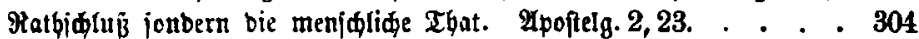

137. 4. Tie Mugeredtigleit bes Tobestutheils über $\delta$. Erröj. Marc. 15, 14. 15306

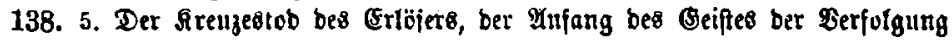

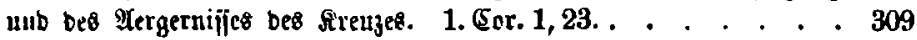

139. 6. Die Entjdulbigung ber unwifienteit gilt feit Eqrifti Tobe nidt mebr jiir bie Sünbe ber Equiften. Pllc. 23,34. . . . . . . . . . 311

140. 7. $3 \mathfrak{n}$ meldem Sinne e8 für unjere umb 9 Inbrex Slinbe eine Entidulbi: gung ber Hnwifienteit giebt. \&uc. 23, 34. . . . . . . . . . 313

\section{Finfte $\mathfrak{B}$ ode. (Sountag 3 ubica.)}

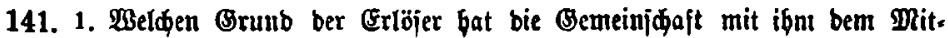
gelteujigten ju verbeifien. \&uc. 23, 43. . . . . . . . . . . 316

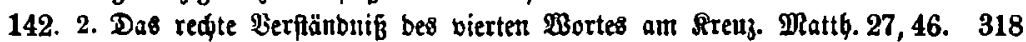

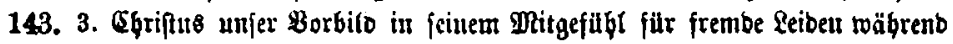
jeines eigenen Seibens. Dlattb. 27,46. . . . . . . . . . . 320

144. 4. Miq bitrftet. $304.19,28$. . . . . . . . . . . . . . 323

145. 5. Unjere Berpflidtung naळ bem Borgange bes Erlöjers ben Befeibigern eben jowohl ફulfe zu leiften als fie von ignen anzunebmen. $304.19,28.325$

146. 6. Bieid bem Erriöjer burfen wir ben Glauben an bas (Bute in ben

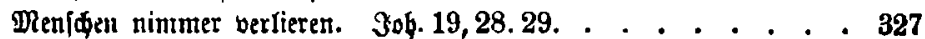

147. 7. E⿺ if volubradt! $304.19,30 .$. . . . . . . . . . . 330

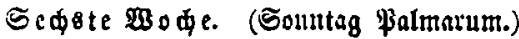

148. 1. Bater in beine bänbe befehle í meinen Beif. \&uc. 23,46. . . . 332

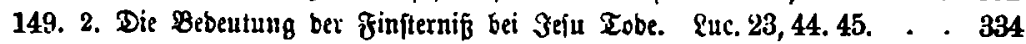

150. 3. Das Berreipen bes Sorbange. Suc. 23,45.. . . . . . . . . 336

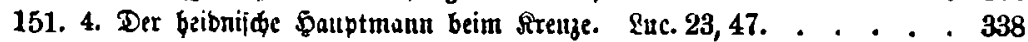

152. 5. Das Bolt unb bie Seinigen beim Areuz bes Erlöjers. \&uc.23,43.49. 339

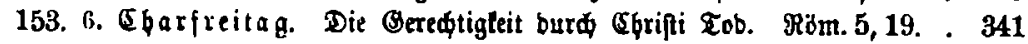

154. 7, Der Zెujanmenbang zwifकen ber Auferfebung b. Ertbjers unb jeinem

Tobe. Upoftelg. 5, 30.31. . . . . . . . . . . . 345 


\section{Die Diterjeit.}

Erfie $B$ o de. Dftern.

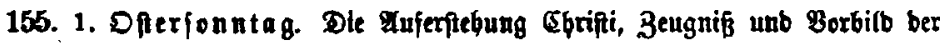
geiftigen suferfesung utferer Tobten. \&uc. 24, 5. 6.

Seite .. .

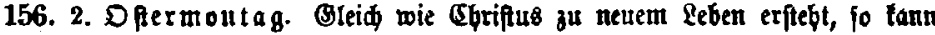
bas Bute uberbaupt nimmer untergeben. Suc. 24,21. . . . . . 351

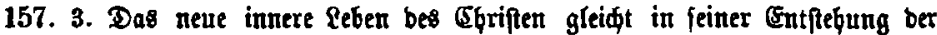
Tuferftebung Ebrift. Möm. 6,4. . . . . . . . . . . . . 353

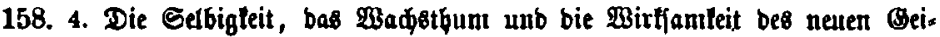

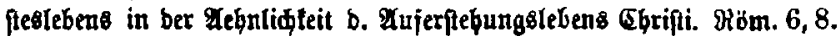

159. 5. Die Berborgenbeit bes neuen geiftigen febent. Col. 3,3. . . . .

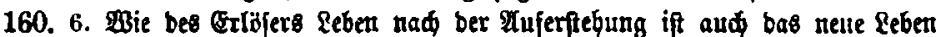
bes Beiftes ojt unterbroden. Эoһ. 7, 37.38. . . . . . . . . 360

161. 7. Die Inufgabe bes neuen geiptigen Lebens bis gur volltommenten Offen= barung beffelben. 1. 3oh. 3,2. . . . . . . . . . . . 362

\section{3ิ weite $\mathfrak{B}$ ode. (Sonntag Quaftmobogeniti.)}

162. 1. Weldes bie Serrliфleit jei, in bie ber Errbjer burd Setben unb Tob eingeben mưfite. Suc. 24, 25. 26. . . . . . . . . . . . . 364

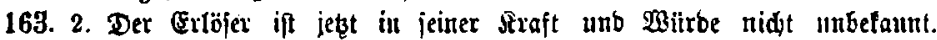
Bbil. 2,9-11. . . . . . . . . . . . . . . . . . .

164. 3. 3n weldem Sinne aud jeß̧t bie \$rebigt von Eqrifto ein Bengnip in

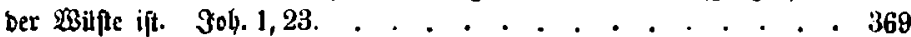

165. 4. Tie Beridiebenteit bes Zెeugnifies vou Eluripto. Pbil. 1, 18. . . . 371

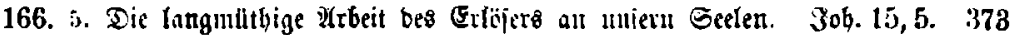

167. 6. Das Mejen ses Meidies (Bottes. Tol.3,3.. . . . . . . . . 375

168. 7. Wer in beun Heide Bottes lei unb wer nid)t. 1. (5or. 12,3. . . . 377

Dritte Bode. (Eountag Diiericorbias Domini)

169. 1. Bon ben 2lemtern im Keide Bottes. 1. (Sor. 12,5. . . . . . . 379

170. 2. Das Berlangen naw bem Seit in (5brifto ift bie einjigi Bebingung jum Eintritt in bas Meidh Bottes. Mpeftely. 11, 17. . . . . . 382

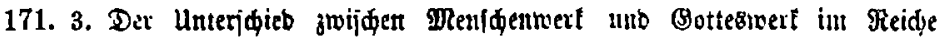
Bottes. Apofielg. 5, 38. 39. . . . . . . . . . . . . . . 384

172. 4. Bas innerbal6 ber driftididen Pirde (3ottes = mo Dienjidentwert jei, barf nidt voreilig entidiebent nuerben. \$ebr. 13,9. . . . . . . 386

173. 5. Die Einlabung zum Meide Bottes joll mit Frentigfeit gefdeben. Pratth. 11, 28-30. . . . . . . . . . . . . . . . . 388

174. 6. Wir fint in Meide Botteo Mtfe Steleute. Mattl. 13,23,. . . . 390

175. 7. Die Beridgiebenbeit ber Menjien in ibren Reigungen uno iquer Ster= lung zun アieidje Botte8. Euc. 17,20.21. . . . . . . . . . 393

Bierte $\mathfrak{B}$ ode. (Somntag Bubilate.)

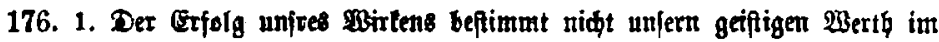
Reide Gotter. Euc 10,20. . . . . . . . . . . . . . 395 
177. 2. Der Gegen Bottes it unabkingig ben Beftrebungen ber MRen= ifen in fement Reid. \$gic 2, 13. . . . . . . . . . . . 397

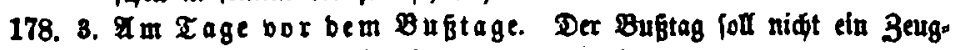
nif̧ bes Griftes bet gurdt fein. 1.301.4, 18.. . . . . . . . 399

179. 4. $\mathfrak{A m} 2 \mathfrak{B}$ istage. Die Bebeutung bes Buftages. Spritub. 14, 34. 401

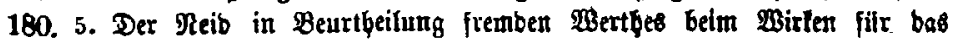
Peid Botter. 1. Tim. 1,5. . . . . . . . . . . . . . 404

181. 6. Die Ungerewtigteit unb Sieblofigltit in ber Bentrţeilung Yuberer allein

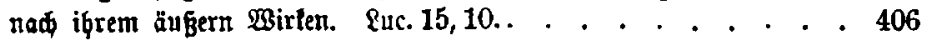

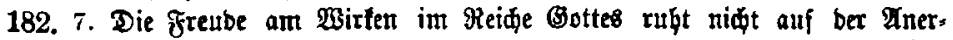
fennung ber Mentiden, joubern auj ben Beivubifein ber gëttlident (3rabe. 2. Sor. $12,9$.

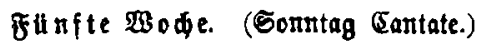

189. 1. Des (Egriften $\mathfrak{B i r f i a m l e i t ~ f u ̈ r ~ b a s ~ \Re e i d ~ G o t t e s ~ i m ~ e n g e r e n ~ u n b ~ m e i - ~}$ teren Ereije. 2. (Eor. 6, 4. . . . . . . . . . . . . 410

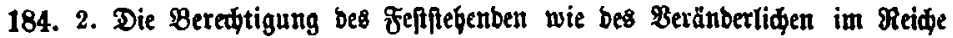
Gotter. פRatth. 9, 17.. . . . . . . . . . . . . . . .

185. 3. Das Shiglingen beffen was für bas gecid Bottes unternommen wor: Den, barf uns nidt ranfelmufitbig madeen. Ser. 10,23. . . . .

186. 4. Die Notbwenbigleit bes Mergernifies, weldes burd bas Böfe im Meidfe Bottes entflebt. Piattb. 18, 7. . . . . . . . . . . . 417

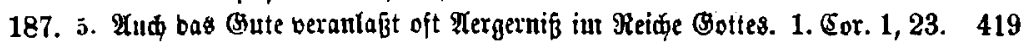

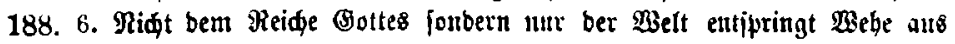
Dem Mergernif. Matţ. 18,7. . . . . . . . . . . . 420

189. $\div$. Die gemeinjame Sdyllb burd/ bas Mergerniß̧. Matth. 18, 7. . . 423

VI. Die Dimmelfahrt $=$ unb Pfingftzeit.

Erfte $\mathfrak{B}$ o

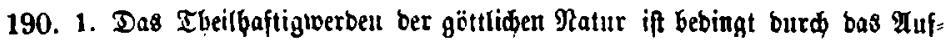
geben ber böjen \&uft. 2. \$etr. 1,4. . . . . . . . . .

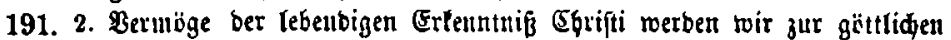
Ratur erboben. 2. \$etr. 1,3. . . . . . . . . . . . . . 427

192. 3. Die emige Errï̈unt. ફebr. 9, 12.. . . . . . . . . . . . 429

193. 4. Unjer $23 a n d e t$ ift im Sgimntel. Bgit. 3,20. . . . . . . . . . 431

194. 5. Şimmelfagrt. Bei bem Sdjeiben beß Ertöjers son ber Erbe trug

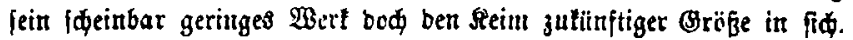
Marc. 4, 31. 32.

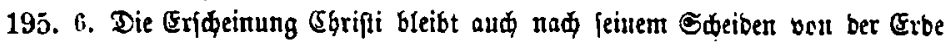

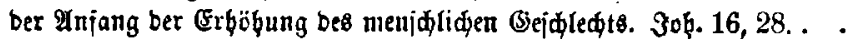

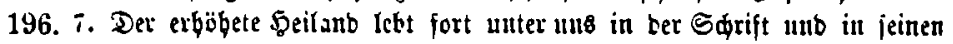
EGenbilberk. Dlattb. 28, 20. . . . . . . . . . . 439

3weite $\mathfrak{B}$ odpe. (Sonntag Erraubi.)

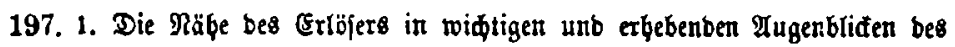
Setens. Mattb. 28, 20. . . . . . . . . . . . . . 
Gite

198. 2. Chrifus ber Pfleger ber beiligen (5üter. 5ebr.8,1.2. . . . . 443

199. 3. Der Unteridieb jwijden bem \$eiligthuu bes alren unb bem geiptigen Tempel bes neuen Bunbes. \$ebr.9,11.12. . . . . . . . . 445

200. 4. Das Rommen Kbrifti zum Geridt. צpoftelg. 1, 11. . . . . . 447

201. 5. Das farjde $\mathfrak{B a r t e n .}$ Ypofielg. 1,4. . . . . . . . . . . . 450

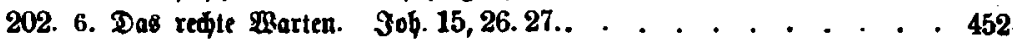

203. 7. Dic $\mathfrak{W a b r b e i t ~ u n b ~ I n g e m e f f e n b e i t ~ b e r ~ g r e f i e n ~ \phi r i f t l i d e n ~ g e f t e . ~}$

(5al. 4, 10. . . . . . . . . . . . . . . . . . . 454

\section{Dritte Bsode. (Pfingften.)}

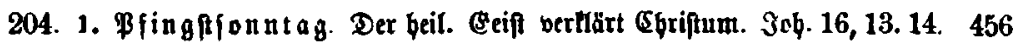

205. 2. Bfingftmontag. Der Friebe (Sbrifti als bie Gabe bes beil. Beiftes.

\$ob. 14, 27. . . . . . . . . . . . . . . . . . . 458

206. 3. In bat Babe bes Beiftes ift alles (Bute zujammengefaft, bas Bott ben Bittenben geben tann. Suc. 11,13. . . . . . . . . . . 461

207. 4. Der Beif erforjdet bie Ziejen ber Bottbeit. 1. (5or. 2, 10.. . . . 463

208. 5. Der Beif ber Belt unt ber Beift aus Bott. 1. Eor. 2.12. . . . 465

209. 6. Der (Beif ber \$raft, ber Siebe unb ber 马udft. 1. Tim. 1, 7. . . . 468

210. 7. Die Unverträgliqfeit bes Beiftes ber Furdit mit bem Beifle ber Rraft, ber Siebe unb ber Budt. 1. Tim. 1,7. . . . . . . . . 470 